\title{
Strategic options for countries to achieve energy for sustainable development
}

\author{
Note by the UNECE secretariat
}

The UN agreed to 17 sustainable development goals that aim to deliver quality of life to all people. The goals cover poverty, health, education, pollution and a range of other objectives. Sustainable development goal number seven is about energy, but all of the goals are underpinned by energy, the golden thread that connects them all. The world also agreed to a set of ambitious climate goals in Paris to limit the rise in global temperatures to well below $2^{\circ} \mathrm{C}$. Unfortunately, the current path is not towards $2^{\circ} \mathrm{C}$ or significantly less than $2^{\circ} \mathrm{C}$ but rather towards much higher levels between $4^{\circ} \mathrm{C}$ and $6^{\circ} \mathrm{C}$. Clearly, addressing the climate challenge is critical and urgent. The climate and development goals can be met only if the energy dimensions are addressed in an integrated way.

An enduring pathway to sustainable development for the UNECE region will integrate both of these perspectives, and energy is the heart of the matter. National circumstances vary significantly, and the choices countries make to meet commitments under the 2030 agenda and the Paris Climate Agreement will not be homogeneous, for example with respect to treatment of fossil energy or nuclear power.

What strategic options do countries have to meet their commitments? Dispassionate choices would be economicsand technology-driven, as the economic order of technology choices to minimize emissions of $\mathrm{CO}_{2}$ while optimizing social and economic benefits can be determined based on local circumstances. In a world driven by such dispassionate choices, invariably improving process and energy efficiency would be the first choice followed by switching to lower carbon fuels and limiting greenhouse gas emissions from higher carbon technology. However, a number of considerations temper any purely economics- and technology-focused policy decisions.

A first consideration is whether or not a country truly is committed to addressing climate change and to attaining the 2030 Agenda's goals. An assumption a priori is that countries intend to respect their commitments to both the 2030 Agenda and the Paris Climate agreement. It will be challenging for countries to deliver on the full slate of objectives to which they have committed, and they will have to make important trade-offs.
A second consideration is the critical role that energy plays in national economies and the sensitivity of governments to modifying that role. The social and economic fabric of many countries or regions within countries are based on activities linked to fossil energy, and deep transformation will have enormous consequences. Beyond employment and the delivery of quality of life, energy can be critical for national incomes, balances of trade, and other geopolitical positioning. Energy market transactions often are disrupted by government actions such as subsidies, sanctions, embargos, or policies regarding self-sufficiency, and actions invariably lead to reactions with long-term consequences.

A third consideration is an extension of the second energy security. Some countries and sub-regions promote energy independence or self-sufficiency as a means to ensure their energy security, while others strive for efficient integration of energy markets. Are countries prepared to collaborate regionally and globally to accelerate attainment of the objectives? Some countries consider that energy supply only can be assured through energy independence and are prepared to pay a premium for it, whereas others consider that energy security can be achieved through diversification of technology choices, suppliers, transit routes, and consumers. Most countries focus on national level actions whereas global and regional solutions would be more effective if there were a culture of trust and reliability in energy transactions. The UNECE region is characterized by a wide range of degrees of trust, so regional business models require a foundation of institutionalized investment and transaction frameworks.

For the UNECE region as a whole, promoting mutually beneficial economic-interdependence would accelerate attainment of the 2030 Agenda through integrative, nexus solutions that the notion of sustainable development offers. For energy, it is critical to think in terms of a wholly interconnected, complex system in which supply, demand, conversation, transport and transmission interact freely and flexibly. Ensuring energy security as part of the ongoing deep transformation creates an imperative to mobilize needed investment in the energy system of the future that is rational 
and pragmatic socially, environmentally, and economically.

However, the concepts of energy security evolved over time from security of supply to a broader view of energy security embracing supply, demand, and transit. However, with increasing penetration of digital technology throughout the energy system and with intensification of climatic events, the energy system is exposed to new risks of either human or natural origins (hacking, terrorist attacks, or natural events like forest fires, hurricanes, and rising oceans). These risks create an added imperative to address the challenge of resilience in terms of both planning and recovery.

A fourth consideration is the timeframe within which countries might take transformational action. The UN intergovernmental panel on climate change has warned there remain only a dozen years for action to limit global warming to a maximum of $1.5^{\circ} \mathrm{C}$, beyond which even half a degree will significantly worsen the risks of drought, floods, extreme heat and poverty for hundreds of millions of people. Urgent and unprecedented changes are needed, but the promise of Paris was matched by recognition that countries will choose their own tempo for change. The data indicate that the world is not on track to meet its commitments. The process of prioritization for some countries could place an early emphasis on development with climate objectives to be pursued later. An enduring element of this consideration will be to mitigate the short-term consequences of actions taken to achieve longer-term objectives.

A fifth consideration is found in the levers of action and the agents of change countries can bring to bear to achieve their commitments in terms of shaping investments and modifying behaviors. The levers of action include market design and structure, network access rules, regulations and regulators, fiscal and monetary policy, taxes and subsidies, monopoly or competition oversight, and the like. The agents of change include the industry players, whether state-owned or private monopolies or large domestic champions on the one hand or a multitude of suppliers, consumers, and new entrants on the other. Governments can influence what will be valid business models through the legal, regulatory, fiscal and contractual frameworks they put in place. Industry will generally deploy their capabilities provided business models are valid (i.e., capital markets will finance them).

The question of business model looms large in this consideration and obliges countries to consider what approaches they are prepared to countenance. Indeed, the choice of business model is one of the defining dimensions for the scenarios developed for this project. One approach is based on large state-owned or state-controlled entities, either regulated monopolies or major players in local, regional, or global markets with significant control over assets and resources. Another approach is that of competitive energy markets wherein natural monopolies such as grids or gas networks are regulated as such, but the rest of the business is considered a competitive marketplace. The textbook definition of a perfectly competitive market is one in which buyers and sellers have no market power, there are no barriers to entry or exit, and there is perfect information. Such a textbook extreme does not exist in practice despite best attempts at market liberalization in many UNECE countries over many years. Variants and blends of the two approaches exist throughout the UNECE region.

A sixth and final consideration is the choice of technologies to deliver energy for sustainable development integrating the three axes of economics, environment, and society. As noted at the outset, national circumstances vary significantly, and countries will make choices that they consider consistent with their national interests while meeting the commitments they have made. The choices made will reflect decisions to include or exclude specific technology and to research and develop new technology. Often the technology options available reflect not only specific national preferences but also the contexts within which policy and investment decisions are to be taken (e.g., the technology choices available to a fossil-rich country with a state-owned monopoly will be very different from those available to a country with a blend of renewable energy and natural gas resources and a culture of market competition).

Given the foregoing considerations, a few strategic approaches emerge:

- Back to the Future: countries can turn to domestic or global energy champions to finance and manage needed investments while deploying an array of policy measures aligned (e.g., standards or fiscal incentives);

- Policy Steroids: countries can extend and deepen current policy approaches with strengthened fiscal and policy commitments; and

- New Business Ecosystems: countries can undertake deeply transformative measures to restructure the energy industry.

\section{Back to the future: energy champions}

Under this strategic approach, countries consider that the urgency of delivering both the 2030 Agenda and the Paris Climate Agreement obliges large-scale, transformative investments but remained concerned with security and reliability. They therefore choose to deploy the technical, financial, and managerial capabilities of large energy companies by:

i. Regulating and enforcing efficiency standards in appliances, buildings, and industry.

ii. Encouraging market concentration and global collaboration among the leading energy companies. Reinforcing networks and extending interconnected operations on a wide regional basis;

iii. Extending support systems for expansion of renewable 
energy capacity including notably in large, centralized facilities with necessary storage or back-up capacity.

iv. Developing a low-carbon transport infrastructure.

v. Deploying carbon capture and storage capacity in line with requirements, supported by policies similar to those deployed for other low or no carbon technology.

vi. Deploying high efficiency low emissions technology to replace the least carbon efficient power technology world-wide.

vii. In countries that accept nuclear power, building new power plants based on existing technology while developing next generation technology.

\section{Policy steroids: stretch}

Under this strategic approach, countries consider that intensification of investments in energy efficiency and renewables and new entrants in both supply and demand sides will accelerate the transformation to a low carbon energy system while meeting the demands of growing economies and populations.

i. Expanding support for low-carbon energy sources, notably renewables, by increasing portfolio obligations and by enabling greater participation of low-carbon distributed generation.

ii. Removing policy barriers to investments in energy efficiency and enhancing investment incentives to accelerate improvements in energy efficiency.

iii. Insisting on policy coherence by removing subsidies that distort energy markets, specifically fossil and enduse subsidies.

iv. Instituting minimum performance standards for fossil fuel using technology (vehicles, power plants)

v. Encouraging accelerated use of ICT (e.g., smart grids and IOT) to improve demand-side participation in energy markets, to improve efficiencies, and to enable greater penetration of intermittent renewables.

vi. Encouraging use of mechanical and chemical storage of electricity.

vii. For countries that accept nuclear power, address capital exposure and improve risk management.

viii. Investing in network infrastructure (gas and electricity) to support penetration of natural gas into a range of end uses (local power generation, natural gas vehicles, back-up support for renewables, heating markets) and to support increased regional penetration of intermittent renewables.

\section{New business ecosystems: deep transformation}

Under this most aggressive strategic approach, countries undertake to transform energy fundamentally. The transformations cover pricing, tariffs, market design, market actors and enabling new categories of players. i. Instituting a real and impactful price on carbon (north of $\$ 120 /$ tonnes $\mathrm{CO}_{2}$, likely revenue neutral but applied world-wide with revenues generated used to support the transition in developing countries).

ii. Investing in major energy infrastructure improvements in transport, power, and natural gas networks, socializing costs and access. Gas infrastructure investments to support penetration of natural gas into a range of end uses (local power generation, natural gas vehicles, back-up support for renewables, heating markets) while reducing methane emissions losses.

iii. Removing all energy subsidies other than those designed to commercialize new technology.

iv. Redesigning energy markets to focus on provision of energy services (thereby making energy efficiency and quality of life improvements the central business model)

v. Deploying ICT widely to improve total system connectivity and efficiencies, improve demand-side participation in energy markets, and enable greater penetration of distributed generation and intermittent renewables

vi. Conceiving balancing markets in the power sector based on energy market boundaries and not political boundaries and enabling full participation by all stakeholders

vii. Deploy and disseminate minimum performance standards throughout the energy system (emissions, power station efficiencies, appliance efficiencies, buildings, and so forth).

\section{Key insights from UNECE's expert groups}

Energy efficiency is a first priority. Before investing in new production and supply infrastructure, countries should improve energy efficiency and productivity when costeffective and, given the enormous potential that exists, should do so at scale in the production, transmission, distribution and consumption of energy. Countries should enable business models that prioritize improving energy productivity.

- Industrial energy efficiency: Reduce energy intensity, improve process efficiencies and rethink technologies that are energy intensive.

- Transport energy efficiency: Rethink large freight transport, deploy electric commuting, and reorganize cities. Introduce renewables into the transport fuel mix (including by transport electrification), energy recuperation, biofuels implementation, city mobility restructuring.

- Building energy performance: Institute and enforce building codes, promote high performance buildings, appliance standards and integrate flexible business models for the residential sector. 
Extend the penetration of renewable energy. Renewable and storage technologies improvements and innovations should be cost effective and made widely available.

- Key innovations and disruptive technologies that will play a crucial role include smart grids, energy storage, renewable heating and cooling. Digital technologies are transforming the power sector.

- Supply and demand-side flexibility is needed in the energy mix for grid resilience and stability.

- Renewable energy potential (power, heat, transport) remains untapped.

Recognise the role that fossil energy will play in a sustainable energy future. The social dimension of the 2030 Agenda cannot be ignored. Though the economic costs are quite high, it is the environmental dimension that is prioritized in the project's modeling results, and yet it is unrealistic that coal will be phased out given the social implications. It may be that there will need to be a better approach to burden sharing so that the social implications of the transition are managed better.

Assumptions, hence outcomes, are tilted toward renewables, away from fossil fuels

- Further dramatic solar and wind cost reductions are a model bias that can be questioned. Similar cost reductions for HELE and CCS also could be supposed.

- Switching (undefined) fossil fuel subsidies to subsidies of renewables is contradictory to creating a level playing field (equal conditions).

- If nuclear power is to be competitive, there is a need to reduce its capital costs. However, there is no evidence that small and medium nuclear reactors will have a lower capital cost [per installed $\mathrm{kW}$ ].

- There is insufficient consideration of storage and hydrogen.

- With advanced technology coal can be clean electric power source: $\mathrm{CO}_{2}$ capture rates can be 99\% now in 2019 with proven technology.

- The scenario analysis must consider the scarcity of certain resources, e.g., rare earths that are indispensable for electric power storage and that could constrain, in particular, EV market expansion.

Natural gas will be key in decarbonisation. The existing gas infrastructure can deliver high storage and transmission capacity in a very efficient and cost-effective way.

- Managing methane emissions from well to burner tip is essential given the global warming potential of methane.

- Decarbonisation projects such as power to gas and energy storage or renewable and low carbon gases implementation (c.f., green/blue hydrogen and biomethane) decrease the environmental impact and carbon footprint of the energy sector.

- Using gas in sectors such as in electricity generation, heating, and transportation mitigates air pollution (including urban air quality).

Given the enduring role of coal, global emissions of methane from the coal sector will continue to increase as mining accesses deeper resources.

- Coal and methane are co-located in many parts of the world. Key coal producing nations often mine coal in gassy regions emitting greater than 750 million tonnes of $\mathrm{CO}_{2} \mathrm{e}$ or 52.5 billion cubic meters of methane per year.

- Methane emissions do not cease at the time of mine closure. Methane escapes through abandoned mine openings (perfect seals are not possible), natural and mining related fractures, and other conduits. Surface mines also emit methane, additional study is needed to verify estimates of emissions.

- Mitigation is possible, and some projects are operating to reduce methane emissions at active and abandoned mines. Annual emissions from one large underground coal mine in the USA can emit 2 million $\mathrm{tCO}_{2}$ e per year or more, and a mitigation project would rival CCS at power plants.

Sustainable Resource Management

- Oil and gas still leading the transition. Renewable energy and nuclear will not displace them without the financial/ policy incentives. Oil should be replaced by gas and renewable energy for heating and transport and kept for petrochemicals.

- Renewable energy alone will not suffice to achieve 1.5/ $2.0^{\circ} \mathrm{C}$ target, gradual phase out of coal and replacement by gas, cleaner coal industry, greater focus on energy efficiency. Contrasting views on nuclear power role. Hydrogen not an effective solution yet. Should seek a sustainable energy mix rather than focusing on particular fuels in an isolated manner.

- Synergies between fossil fuels and renewable energy. Increasing role of NG as a bridging fuel leading to 2030.

- Contrasting views on CCS: Viable technology if costs decrease. Huge investments required, which may have a negative social impact, currently no policy incentives provided. CCS and bio-energy with CCS likely not to be ready even by 2050 .

- Massive amounts of critical elements required (e.g. batteries, PVs), which could be a limiting factor also due to geopolitical differences; however, alternative technology, acceptable international standards, efficient use and circular economy may address it.

- Business models and disruptive technologies: Shale oil disruptive force in recent years, new nuclear technologies (such as molten salt reactors) thorium, better storage technologies, decentralized renewable energy production 


\section{Policy recommendations}

Commit to minimizing adverse energy system impacts on climate, ecosystems and human health. Governments should develop new, transformative policy instruments and speed up regulatory reform.

\section{Energy market reform}

1. Design energy markets as a holistic system

2. Prices should reflect full costs: provide durable price signals on GHG emissions.

3. Ensure reliability, access, stability; deploy 1-t strategic energy planning.

4. Encourage new business models based on competitive low-carbon energy products and services

5. Alternative business models moving away from energy as commodity (push model/customer communication as a one-dimensional bill) to energy as true utility (service/public good?) where customer partners with (or even replaces) the provider.

6. Consider interplay of technologies, including between flexible clean coal, natural gas and renewables, and encourage enhanced engagement of consumers in market design by enabling distributed generation.

7. Incentives for energy efficiency, renewable energy, clean tech

8. Limit subsidies to overcoming s-t obstacles to commercialize

9. Reduce carbon intensity

Energy Efficiency:

Governments need to commit to look at investing in EE before any investments in production and supply infrastructure. Set clear targets for each country to reduce energy consumption for $15 \%$ in 2050 compared to 2020

10. Enable energy efficiency investments — develop business models with focus on increased energy efficiency and savings (negawatt pricing)

11. Sell services, not products

12. Industry sector: Set targets for energy intensity, phaseoff inefficient technologies, obligatory EMS and increase R\&D for energy intensive technologies.

13. Transport sector: Phase-off all oil vehicles by 2050 , phase-off all public transportation on oil by 2030, increase R\&D for large freight transport and develop progressive vehicle standards.

14. Buildings sector: Mandatory new buildings to be $40 \mathrm{kWh} / \mathrm{m}^{2} /$ year by 2035 and NZEB by 2050 , all public buildings to be NZEB by 2035, all energy intensive appliances to be programable based on open standards framework.

15. Urban energy efficiency planning

16. Minimum energy performance Standards
17. Energy efficiency education

Renewable energy

18. Set clear short-term and long-term targets for each country to increase share of renewable energy in energy systems.

19. Laws \& taxes appropriately modified to foster the rapid demand and supply of renewable energy business models: Focus on PPP and public based investments, ESCO scheme combined with energy efficiency. Distributed RES generation and microgeneration (prosumers), microgrids.

20. Transport sector: Increase share of electric, bio fueled, fuel celled vehicles.

21. Industry sector: Use of local renewable sources, waste energy utilization.

22. Mandatory new buildings to be equipped with PV construction materials, solar water heaters and heat pumps, energy storage devices and load management systems.

23. Full grid access and reinforcement

24. Market reform for supply/demand flexibility, storage, energy efficiency, and efficient transmission and distribution

25. Promote decentralized direct use of renewable energy to provide on-site energy services.

26. R\&D collaboration on energy efficiency and renewable energy

27. Recognise the importance of renewable gases (such as hydrogen and biomethane). Sector coupling and sectoral integration are essential elements for decarbonisation.

\section{Fossil Fuels}

28. Encourage member States to pass legislation that clarifies ownership and promotes global recognition that coal-associated methane is an important energy resource and a powerful greenhouse gas. This will enable beneficial use and mitigate emissions.

29. Encourage preferential access to pipelines and power grids for gas and electricity produced from methane sourced at active and abandoned coal mines.

30. Natural gas and electricity pricing should be preferential for energy produced from coal mine methane (e.g., feed-in tariffs. Introduce tax incentives to support CMM projects or tax carbon emissions.

31. Emissions trading groups should be formed between coal mines and consumers to promote methane emissions reductions at coal mines through projects financed by coal consuming industry, i.e, power, cement and steel producers and used as offsets for consumer's emissions. 
32. Support finance of coal mine methane emissions reductions projects.

33. Introduce carbon dioxide reduction and minimum efficiency standards for electric power generation from coal, natural gas, fuel oil, and biomass.

34. Ensure a cost-efficient energy storage system for the short, medium and long-term. In this regard, the role of gas system should be acknowledged. Via Power-togas technologies electricity can be stored in the form of gas within the gas system at minimum costs. Promote the utilization, repurposing and optimization of the gas infrastructure. The gas system can deliver high storage and transmission capacities in a very efficient and cost-effective way. Leveraging gas infrastructure should be considered as a point of departure for any energy transition pathway.

35. Management of methane emissions across value chains should enable the role of gas in the future global energy mix by helping governments achieve their climate goals, instilling stakeholder confidence with respect to gas' environmental value and term predictability that allow industrial planning and investment

\section{Energy Access}

36. Mobilize resources to finance access

37. Promote the necessary energy (gas and electricity) infrastructure and energy interconnectivity among markets to enabling energy supply competition, liquidity, price convergence, diversification of energy supplies, renewable integration, etc., resulting in more competitive and affordable prices for consumers

38. Promote QOS regulation

39. Improve performance of urban environments through smart urban ecosystems.

\section{Energy Security}

40. Standards for interconnections, interoperability and trading

41. Regional infrastructure projects

\section{Sustainable resource management}

42. Global governance required to ensure optimal usage of scarce/limited resources. Transition from a linear to a circular model. EU Waste Directive should promote further this shift. Waste management part of the initial development plans. Zero waste principal should be built into incentives. Adoption of innovative accounting tools such as the System of Environmental-Economic Accounting (SEEA).

43. Resource curse can be avoided if a dynamic systems approach and proper information architecture are used in studying the problems, proper tools could be developed. The UN Resource Management System is one such approach.

\section{Finance and investment}

44. Align investment incentives with the 2030 Agenda

45. Invest in flexibility (supply, grid, and demand)

46. Involve banks to create financial products for bankable energy projects

47. Establish investment framework conditions for efficient provision of energy services

48. Investment-friendly environment (transparent energy strategy and mechanism for fixing renewable energy targets, mitigation of currency devaluation risk, affordable interest rates, availability of long term and cheap financing, regional cooperation, business support to research activities aimed at rational use of energy resources and development of new technologies, feed-in tariffs and other incentives are desired).

49. Deploy tools to reduce the perceived risk of energy efficiency and renewable energy projects

50. Create financing instruments designed for noninstitutional end users.

51. Introduce standard on financing carbon capture, utilization and storage projects, and commit to develop CCS: fossil fuels-based generation can be cleaner subject to systems approach and proper investments.

\section{Technology}

52. Pathways should be technology-neutral and always the most cost-efficient, bearing in mind that the energy future under COP21 agreement will be a combination of renewable/low carbon electrons and renewable/low carbon molecules

53. Support RD\&D of new technology

54. Acceptable international standards - recycling and circular economy should address increased demand for resources extraction (e.g. rare earth elements used in permanent magnet generators or lithium for batteries).

55. Development of storage technologies should be pushed by governments as strongly as possible, main game changer for RE. Heat storage in molten salts and other forms of storage should also be investigated.

56. Role of nuclear filling the gap of reliable, low carbon electricity may be considered. In many countries, consultations with governments have already determined that nuclear has to be a key component especially if uranium and possibly thorium resources available from the country.

57. The transport sector, particularly rail and maritime should be pushed by governments as large carbon contributors not bound by government legislation. 